\title{
A brief review of (multi-scale) modelling approaches to segregation
}

\author{
Anthony Thornton ${ }^{1, \star}$ \\ ${ }^{1}$ Department of Thermal and Fluid Engineering, MESA+, University of Twente, NL.
}

\begin{abstract}
Segregation in dense granular flows is a large problem in many areas of industry and the natural environment. In the last few years an advection-diffusion style framework has been shown to capture segregation in many geometries. Here, we review the different ways such a framework has been obtained by different authors, compare the forms and make recommendations for the best form to use. Finally, we briefly outline some of the remaining open-questions.
\end{abstract}

\section{Introduction}

Segregation of dense granular free-surface flows is easy to observe in our daily lives, in nature and in industry. In nature, segregation has a massive effect on the dynamics of large-scale particulate flows, e.g. landslides, avalanches and pyroclastic flows [1-4] and even bed-load transport [5]. In an industrial setting, segregation almost always occurs when material is transported from one point to another. Hence segregation is an issue in transport chutes $[6,7]$, rotating drums $[8,9]$, hoppers $[10,11]$, transfer point (heap formation) [12], coaters [13], die filling [14] and even mixers [15]. Almost all bulk-handling industries have to deal with segregation issues from food to pharma and even steel-making.

In this paper we present a continuum framework which has been used to describe segregation due to both differences in size and density in dense granular flows. This framework contains free functions and we discuss different ways of determining these functions to close the framework, resulting in a predictive model.

\section{The segregation framework}

The framework is normally presented as an advectiondiffusion equation of the form,

$$
\frac{D \phi}{D t}-S_{r} \frac{\partial}{\partial z} F[\phi]=\frac{\partial}{\partial z}\left(D[\phi] \frac{\partial \phi}{\partial z}\right)
$$

where $\frac{D}{D t}=\frac{\partial}{\partial t}+\mathbf{u} \cdot \nabla$ is the material derivative, $\mathbf{u}=(u, v, w)$ is the bulk velocity with components in $x-, y-, z-$ directions respectively and $\phi$ is the granular volume fraction of smaller/denser species [16-18]. The segregation flux function $F(\phi)$ and the diffusion function $D(\phi)$ need to be determined in order to close the model. They are determined by either modelling or numerical simulation of the dynamics at the particle level; hence in some sense this is a multi-scale model for segregation.

^e-mail: a.r.thornton@utwente.nl

A video is available at https://doi.org/10.48448/j47v-9z31
Written in the form (1) it is implicitly assumed that both segregation and diffusion only act in the $z$-direction which is often taken to be (almost) aligned with the direction of gravity. This is clearly an approximation and in reality both $F$ and $D$ should be vectors. To-date there has been very little work on what exactly sets the direction of segregation. However, the diffusion is expected to be isotropic and hence the direction should be given by the gradient of $\phi$ i.e. $\nabla \phi$.

It is common to take a simple constant for the diffusion term i.e. $D[\phi]=D_{r}$. This assumption has been tested with discrete particle simulations and shown that it matches the general trends but does not get the details right and the fit lies outside the $95 \%$ confidence interval [19]. Various authors have measured the ratio of the magnitude of segregation to diffusion, the so-called, segregation Péclet number and report values in the range 3-19; for examples see $[19,20]$. For these reasons, in the remainder of this paper we will not consider the diffusion and take $D \equiv 0$.

\section{Deriving the framework}

Originally, the framework (1) was derived using mixture theory $[16,17]$. Mixture theory is popular in certain fields to describe complex mixtures and allows one phase to turn into another [21].

The key idea of mixture theory is to assume that every point is simultaneously occupied by all phases which in our case is two granular species and the interstitial fluid (void space) between these. Note, here we are restricting our attention to two distinct granular phases; however, extensions to fully polydispersed mixtures [6] and $n$ distinct species [22] do exist. Mixture theory then allows overlapping partial density, $\rho^{v}$, partial velocity, $\mathbf{u}^{v}$, and partial stress, $\sigma^{v}$, fields to be defined for each constituent $v$.

In mixture theory each constituent, $v$ must satisfy partial mass

$$
\frac{\partial \rho^{v}}{\partial t}+\nabla \cdot\left(\rho^{v} \mathbf{u}^{v}\right)=0
$$


and momentum

$$
\rho^{v} \frac{D_{\nu} \mathbf{u}^{v}}{D t}=-\nabla \boldsymbol{\sigma}^{v}+\rho^{v} \mathbf{g}+\boldsymbol{\beta}^{v},
$$

balance equations. Here, $D_{v} / D t=\partial / \partial t+\boldsymbol{u}^{\nu} \cdot \nabla$ is the total derivative moving with constituent $v$ and $\rho^{v} \boldsymbol{g}$ is the gravitational force. The interaction drag, $\boldsymbol{\beta}^{\nu}$, is the force exerted on phase $v$ by the other constituent. By definition, the sum over all the constituents is equal to zero, i.e. $\sum \boldsymbol{\beta}^{v}=\mathbf{0}$. The bulk density, $\rho$ and the bulk stress, $\sigma$, are by construction the sum of the partial densities and partial stresses.

To close mixture theory the relationship for partial stress and the interaction drags must be specified. For segregation problems the following four assumptions are normally enforced

1. The bulk flow is incompressible

2. Normal acceleration terms are negligible

3. The interaction drags are Darcy like [16] i.e.

$$
\boldsymbol{\beta}^{v}=\sigma \nabla f^{v}-\rho^{v} c\left(\boldsymbol{u}^{v}-\boldsymbol{u}\right),
$$

4. A non-linear stress partitioning is introduced [7] i.e.

$$
f^{v}[\phi]=\frac{\sigma_{z z}^{v}}{\sigma_{z z}} .
$$

Here $f^{v}$ is the over/under stress, which indicates the amount of excess stress borne by the larger/smaller particles and is a convenient mathematical construct. This difference in stress between the different granular species has been measured in particle simulations by many authors (see e.g. [23-25]); however, in order to get consistent answers, care has to be taken in how to share the forces between the different particles; discussed more later [26]

The physical origin of this over/under stress is currently an open research area. Originally authors argued it was a combination of non-preferential squeeze expulsion and size-preferential kinetic sieving [16, 27]. However recently, people have suggested new physical mechanisms for the rise of large particles and this remains an active research area [28-32].

Applying the four assumptions (and closure (4), (5)) to the mixture theory framework (2), (3) results in the segregation framework (1). Full details of the derivation can be found in many places e.g. [16]. The following key relationships follow from the derivation

$$
F=\left(f^{s}-\phi\right) / c \frac{\partial \sigma_{z z}}{\partial z}
$$

and

$$
w^{s}=w-\frac{F}{1-\phi}
$$

where $w^{s}$ is the $z$-component of the velocity of the small/heavy species and $w$ the bulk velocity $z$-component.
Table 1. Comparison of different approaches. NM-Not mixture theory; whereas numbers indicate how a mixture theory model was closed. Note, each has a free parameter here called $S_{r}$ however this is not the same parameter.

\begin{tabular}{cccc}
\hline Type & Model & $\boldsymbol{F}$ & $\boldsymbol{D}$ \\
\hline NM & BW [33] & $-S_{r} \phi(1-\phi)^{2}$ & $D_{r}$ \\
NM & DU [34] & $-S_{r} \phi(1-\phi)$ & $D_{r}$ \\
1 & GT [16] & $-S_{r} \phi(1-\phi) \rho g$ & 0 \\
1,4 & Hill [35] & $-S_{r}(\phi(1-\phi)) \frac{1}{\rho} \frac{\partial \sigma_{z z}^{k}}{\partial x}$ & $D_{r}$ \\
1,4 & Marks [6] & $S_{r} g \dot{\gamma}(\hat{s}-\hat{\rho})\left[\frac{\phi(1-\phi)}{\phi+(1-\phi) \hat{s}}\right]$ & $D_{r}$ \\
1,3 & Chassange [36] & $S_{r}(\hat{s}-1) I^{0.81} \phi(1-\phi)$ & $D_{r}$ \\
1,2 & GG [37] & $-S_{r} \phi(1-\phi)(1-\kappa \phi) \rho g$ & 0 \\
3 & TT [7] & $S_{r} g \dot{\gamma}\left(\hat{s}^{3}-\hat{\rho}\right)\left[\frac{\phi(1-\phi)}{\phi+(1-\phi) \hat{s}^{3}}\right]$ & 0 \\
3 & Schlick [38] & $S_{r} d_{s} \ln \left(\frac{d_{l}}{d_{s}}\right) \dot{\gamma} \phi(1-\phi)$ & $D_{r}$ \\
4 & TWT [25] & $S_{r} \phi(1-\phi)(1-\kappa[\hat{s}] \phi) \frac{1}{\rho} \frac{\partial \sigma_{z z}^{k}}{\partial x}$ & 0 \\
\hline
\end{tabular}

\section{Closing the framework}

In the previous section we outlined how the framework is normally derived; however, it should be noted that various models of segregation that pre-date this derivation are also contained within the general advection-diffusion framework (1) i.e. models by Bridgewater et al [33] and Dolgunin and Ukolov [34].

There have been many authors (and groups) to work with this segregation framework, here we try and review all the different approaches. There has been broadly four approaches to closing this framework:

1. Postulating a closure for the stress;

2. Experimentally measuring percolation velocities;

3. Using particle simulations to compute the segregation-flux;

4. Using particle simulations to compute the stress closure.

Table 1 summarises some of the approaches taken and the resulting obtained flux. Next we will briefly review each of these proposed fluxes.

In the original derivation of the framework of Gray and Thornton only two different size particles, small (s) and large (1) were considered [16]. Also the model was closed by deriving the simplest (via a Taylor series expansion) possible form for the stress partition that obey simple physics constraints, i.e.

$$
f^{s}=1-b(1-\phi) \text { and } f^{l}=1+b \phi
$$

where $b$ is to be determined constant. Shortly afterwards Gray and Chugunov [18] added a simple diffusion term to the mixture theory model resulting in the same form as Dolgunin and Ukolov [34].

One of the key differences with mixture models to earlier models is it explicitly follows that segregation is driven by gradients in stress. Originally the stress in the material was assumed to be lithostatic, therefore the gradient of 
stress is simply $\rho g[16,17]$. Fan and Hill [35] theoretically showed there is a second contribution to the stress caused by velocity fluctuations i.e. the kinetic stress and showed this should also lead to segregation. This was shown to be active in vertical chutes (i.e. no gravity). Since the kinetic stress mechanism was proposed, it has by many authors, been shown to be the dominant mechanism, even in drums [24] and inclined planes [25]. Also in all these geometries the direction of both mechanisms are the same; therefore there has been some debate about whether there are one or two mechanisms. We will not present the results here, but it is the opinion of this author that for spherical particles, the kinetic stress mechanism is dominant; however, for non-spherical particles both mechanisms are important and can act in different directions causing changes in the segregation direction.

Marks et al [6] were the first to extend the model to polydispersed particle sizes, to include density differences and to introduce first principle physics, albeit inspired by earlier particle simulations, to the model. Their resulting flux (simplified for two components) can be found in table 1 where $\hat{s}$ is size ratio, $\hat{\rho}$ is the density ratio and $\dot{\gamma}$ is the shear rate. However, it should be noted, the model is not fully closed as they still have one free parameter coming from the drag law (4). Whereas Chassange et al [36] used particle simulations to obtain a modified flux for bed-load transport which depends on the inertia number $I$ and the size-ratio, $\hat{s}$.

Gajjar and Gray [37] considered analytical solutions for a class of cubic fluxes and shortly after Van der Vaart et al [39] used the velocity relation (7) to experimentally measure these forms in a shear box. Later, the cubic flux was shown in both particle simulations and experiments to capture the leading order features of segregation patterns on a moving belt [40, 41].

Several authors have used particle simulations to directly determine the segregation flux $F$. By computing, in particle simulations, situations where size-density balance resulting in zero segregation in a wide range of different systems, Tunuguntla et al [7] suggested a modification of the flux of Marks et al [6] raising the power of size ratio to 3 i.e in this version it is volume ratio not size ratio that sets the strength of segregation. This flux leads to terms which are similar to buoyancy forces which is interesting as a lot of recent microscopic models [31,32] suggest there may be a modified buoyancy style lift force on the large particles. Whereas Schlick et al [38] used a different approach with particle simulations to obtain a form which implicitly depends on both the size of the small $d_{s}$ and large particles $d_{l}$; however, this also still has one extra free parameter.

Weinhart et al [23, 26] developed a new method of extracting the particle stress fractions, $f^{v}$, based on coarse graining e.g. [42]. This method has many advantages over previous methods, here, we will just emphasise one: by construction it gives fields which are compatible with the theory.

Tunuguntla et al [26] showed various important results with the new method. Firstly, due to the property stated above, they showed the only correct way to distribute the force of a mixed contact is in proportion to length of the

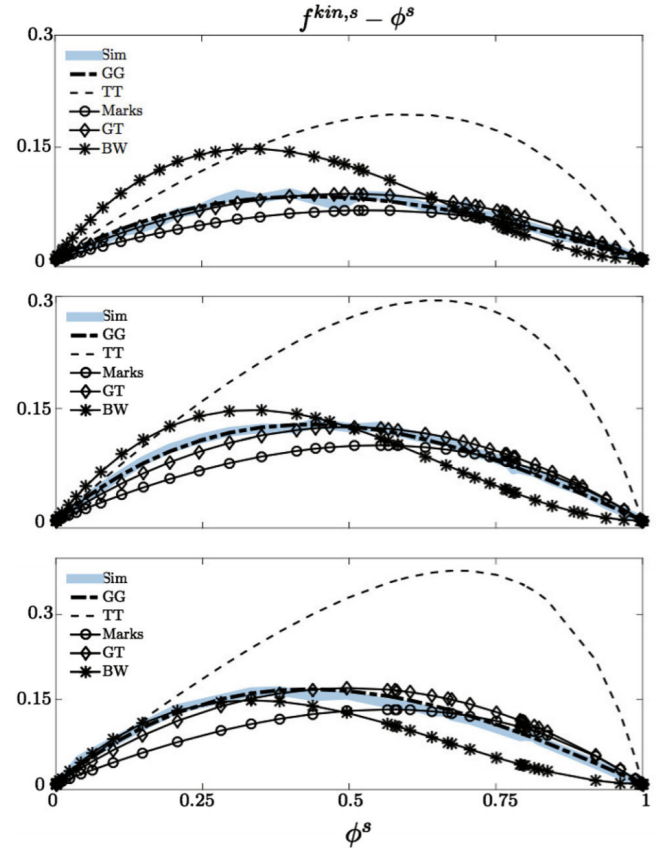

Figure 1. Comparison of different $f$ for different binary size ratios (1.3, 1.5 and 1.7). The blue lines are coarse-grained fields from MercuryDPM [43] simulations. See table 1 for models names for associated $F$ s. Figure reproduced from fig. 10 of [25]. Note, in the original figure, the $\mathrm{x}$-axis is incorrectly labelled so please see the erratum.

branch vector within the particle. With traditional binning based methods, there is a free parameter in how you split the force of a contact between two different species. Unfortunately this is not actually a free choice and only one results in the correct answer.

Secondly, it allowed them to extract fields from timedependent (segregating) not steady-state (segregated) systems [25]. Showing both gives the same answer: this is a key, but boring, result as previously most results have been obtained only from steady-state data.

Thirdly, it confirmed previous results that the (over/under) contact stress is very small and probably not responsible for segregation; whereas the kinetic stress has a strong effect [23-25].

Finally, it allowed the computation of the 'correct' stress partitioning function [25]. They concluded the following form was best

$$
F[\phi]=-A \phi(1-\phi)(1-\kappa[\hat{s}] \phi) \frac{1}{\rho} \frac{\partial \sigma_{z z}^{k}}{\partial x}
$$

which is a combination of many of the previous ideas. It is driven by the kinetic stress [35], has the shape suggested by [37] but the degree of asymmetry is size dependent. However, it is unclear if the size dependence is as suggested by Marks et al [6], Tunuguntla et al [7] or Schlick et al [38]; as the authors only provided the values of $\kappa$ for three cases and did not provide a fit of $\kappa$ as a function of $\hat{s}$. 


\section{Discussion and conclusion}

We have reviewed different approaches to modelling segregation using an advection-diffusion style framework i.e. (1) and have shown this approach can be quite successful in describing segregation in complex flows. However, we have not explicitly shown it here, but closing via the stress partition, $f$, or directly the flux, $F$, leads to a different final form for $F$ [25]. This implies there is something wrong with the assumed drag law i.e. (5). To-date there has only been limited research on this [23]. In future, more attention should be paid to the drag law, how the diffusion is included in this framework and how to calibrate for real materials.

\section{References}

[1] R.M. Iverson, M. Logan, R. LaHusen, M. Berti, J. Geophys. Res. Earth Surf.: 115, 121 415-441 (2010)

[2] M. Woodhouse, A.R. Thornton, C. Johnson, P. Kokelaar, J.N.M.T. Gray, J. Fluid Mech. 709, 543 (2012)

[3] C.G. Johnson, B.P. Kokelaar, R.M. Iverson, M. Logan, R. Lahusen, J. Gray, J. Geophys. Res. 117, F01032 (2012)

[4] I.F.C. Denissen, T. Weinhart, A. te Voortwis, S. Luding, J.M.N.T. Gray, A.R. , Thornton, J. Fluid Mech. 866, 263-297 (2019)

[5] P. Frey, H. Lafaye de Micheaux, C. Bel, R. Maurin, K. Rorsman, T. Martin, C. Ducottet, Adv Water Resour. 136, 103478 (2020)

[6] B. Marks, P. Rognon, I. Einav, J. Fluid Mech. 690, 499 (2012)

[7] D.R. Tunuguntla, O. Bokhove, A.R. Thornton, J. Fluid Mech. 749, 99 (2014)

[8] K.M. Hill, J. Kakalios, Phys. Rev. E 49, R3610(R) (1994)

[9] C.R.K. Windows-Yule, B.J. Scheper, A.J. van der Horn, N. Hainsworth, J. Saunders, D.J. Parker, A.R. Thornton, New J. Phys. 18, 023013 (2016)

[10] W.R. Ketterhagen, J.S. Curtis C.R. Wassgren, A. Kong, P.J. Narayan, B.C. Hancock, Chem. Eng. Sci. 62, 6423 (2007)

[11] H. Xiao, Y. Fan, K.V. Jacob, P.B. Umbanhowar, M. Kodam, J.F. Koch, R.M. Lueptow, Chem. Eng. Sci. 193, 188 (2019)

[12] Y. Fan, Y. Boukerkour, T. Blanc, P.B. Umbanhowar, J.M. Ottino, R.M. Lueptow, Phys. Rev. E 86, 051305 (2012)

[13] J. Li, C. Wassgren, J.D. Litster, Chem. Eng. Sci. 95, $203(2013)$

[14] Y. Guo, C.Y. Wu, K. Kafui, C. Thornton, Powder Technol. 206, 177 (2011)

[15] T. Shinbrot, A. Alexander, F.J. Muzzio, Nature 397, 675 (1999)

[16] J.M.N.T. Gray, A.R. Thornton, Proc. Royal Soc. A 461, 1447 (2005)

[17] A.R. Thornton, J.M.N.T. Gray, A.J. Hogg, J. Fluid Mech. 550, 1 (2006)
[18] J.M.N.T. Gray, V.A. Chugunov, J. Fluid Mech. 569, 365 (2006)

[19] A.R. Thornton, T. Weinhart, S. Luding, O. Bokhove, Int. J. Mod. Phy. C. 23 (2012)

[20] D.V. Khakhar, J.J. McCarthy, J.M. Ottino, Chaos 9, 594 (1999)

[21] L. Morland, Surv. Geophys. 13, 209 (1992)

[22] J.M.N.T. Gray, C. Ancey, J. Fluid Mech. 678, 535 (2011)

[23] T. Weinhart, S. Luding, A.R. Thornton, From discrete particles to continuum fields in mixtures, in Powders and grains (2013), Number 1542 in 1202

[24] K.M. Hill, D.S. Tan, J. Fluid Mech. 756, 54 (2014)

[25] D.R. Tunuguntla, T. Weinhart, A.R. Thornton, Comput. Part. Mech. 4, 387 (2017)

[26] D.R. Tunuguntla, A.R. Thornton, T. Weinhart, Comput. Part. Mech. 3, 349 (2016)

[27] S.B. Savage, C.K.K. Lun, J. Fluid Mech. 189, 311 (1988)

[28] F. Guillard, Y. Forterre, O. Pouliquen, J. Fluid Mech. 807, R1 (2016)

[29] L. Staron, J.C. Phillips, Comput. Part. Mech. 3, 367 (2016)

[30] L. Jing, C.Y. Kwok, Y.F. Leung, Phys. Rev. Lett. 118, 118001 (2017)

[31] K. van der Vaart, M.P. Schrojenstein Lantman, T. Weinhart, S. Luding, C. Ancey, A.R. Thornton, Phys. Rev. Fluids (2018) 3, 074303

[32] L. Jing, J.M. Ottino, R.M. Lueptow, P.B. Umbanhowar, Phys. Rev. Research 2, 022069 (2020)

[33] J. Bridgwater, W. Foo, D. Stephens, Powder Technology 41, 147 (1985)

[34] V.N. Dolgunin, A.A. Ukolov, Powder Technol. 83, 95 (1995)

[35] Y. Fan, K.M. Hill, New J. Phys. 13, 095009 (2011)

[36] R. Chassagne, R. Maurin, J. Chauchat, J.M.N.T. Gray, P. Frey, (2019), arXiv: 1909.07687 (2020)

[37] P. Gajjar, J.M.N.T. Gray, J. Fluid Mech. 757, 297 (2014)

[38] C.P. Schlick, A.B. Isner, B.J. Freireich, Y. Fan, P.B. Umbanhowar, J.M. Ottino, R.M. Lueptow, J. Fluid Mech. 797, 95 (2016)

[39] K. van der Vaart, P. Gajjar, G. Epely-Chauvin, N. Andreini, J.M.N.T. Gray, C. Ancey, Phys. Rev. Lett. 114, 238001 (2015)

[40] P. Gajjar, K. van der Vaart, A.R. Thornton, C.G. Johnson, C. Ancey, J.M.N.T. Gray, J. Fluid. Mech. 794, 460 (2016)

[41] K. van der Vaart, A.R. Thornton, C.G. Johnson, T. Weinhart, L. Jing, P. Gajjar, J.M.N.T. Gray, C. Ancey, Granul. Matter 20, 46 (2018)

[42] T. Weinhart, A.R. Thornton, S. Luding, O. Bokhove, Granul. Matter 14, 289 (2012)

[43] T. Weinhart, L. Orefice, M. Post, M.P. van Schrojenstein Lantman, I.F. Denissen, D.R. Tunuguntla, J. Tsang, H. Cheng, M.Y. Shaheen, H. Shi et al., Comput. Phys. Commun. 249, 107129 (2020) 